\title{
Cylindrical Inclusion Protein of Wheat Yellow Mosaic Virus Is Involved in Differential Infection of Wheat Cultivars
}

\author{
Takehiro Ohki, ${ }^{1, \dagger}$ Takahide Sasaya, ${ }^{2}$ and Tetsuo Maoka ${ }^{1}$ \\ ${ }^{1}$ Division of Agro-Environmental Research, Hokkaido Agricultural Research Center, NARO, Hitsujigaoka, Toyohira, Sapporo, Hokkaido \\ 062-8555, Japan \\ 2 NARO Headquarters, 3-1-1 Kannondai, Tsukuba, Ibaraki 305-8517, Japan \\ Accepted for publication 28 March 2019.
}

\begin{abstract}
Wheat yellow mosaic virus (WYMV) belongs to the genus Bymovirus in the family Potyviridae and has a bipartite genome (RNA1 and RNA2). WYMV in Japan is classified into three pathotypes (I to III) based on its pathogenicity to wheat cultivars. Among these three, pathotypes I and II are discriminated by their pathogenicity to the wheat cultivar Fukuho; pathotype I infects Fukuho but pathotype II does not. In the present study, the genomic regions that are involved in such pathogenicity were examined using infectious viral cDNA clones of pathotypes I and II. Reassortant experiments between viral RNA1 and RNA2 revealed the presence of a viral factor related to pathogenicity

Furthermore, analysis of chimeric and site-directed mutants revealed that three amino acids at the $\mathrm{N}$-terminal region of CI protein were involved in pathogenicity to Fukuho. On the other hand, at the singlecell level, pathotype II replicated in protoplasts of Fukuho similar to that of pathotype I virus. These data suggest that differential pathogenicity between pathotypes I and II was considered to depend on the ability of cell-to-cell or long-distance viral movement, in which CI protein is involved. To the best of our knowledge, this is the first report to show the involvement of the bymoviral CI protein in pathogenicity.
\end{abstract} in RNA1. A chimeric pathotype II virus harboring a cylindrical inclusion (CI) cistron from pathotype I facilitated systemic infection of Fukuho, indicating that CI protein is involved in pathogenicity.
Keywords: bymovirus, pathotype, systemic infection, virology, wheat cultivar
Wheat yellow mosaic virus (WYMV) is found in Japan and China, and causes yellow mosaic and dwarf symptoms in wheat (Triticum aestivum L.) (Han et al. 2000; Inoue 1969). WYMV belongs to the genus Bymovirus in the family Potyviridae and is transmitted by the plasmodiophorid Polymyxa graminis (Adams et al. 2012). The virions are flexuous filaments with two modal lengths (approximately 550 and $275 \mathrm{~nm}$ ), with a diameter of $13 \mathrm{~nm}$ (Inoue 1969). They contain two linear, positive-sense, single-strand RNAs (Namba et al. 1998). RNA1 is approximately $7.7 \mathrm{~kb}$ long and encodes a polyprotein that contains eight putative proteins: $\mathrm{P} 3,7 \mathrm{~K}$, cylindrical inclusion protein $(\mathrm{CI}), 14 \mathrm{~K}$, genome-linked viral protein (VPg), protease (Pro), RNA-dependent RNA polymerase (NIb), and a coat protein $(\mathrm{CP})$. A small open reading frame resulting from a frameshift in P3 cistron, termed P3N-PIPO (Chung et al. 2008), is also found in RNA1 of WYMV. RNA2 is approximately $3.7 \mathrm{~kb}$ long and encodes a polyprotein that contains a $28-\mathrm{kDa}$ protein $(\mathrm{P} 1)$ and a 73-kDa protein (P2).

In previous studies on the biological and genetic diversity of WYMV in Japan, WYMV was divided into three pathotypes based on their ability to infect three wheat cultivars: pathotype I infects Fukuho and Nambu wheat but not Hokkai 240, pathotype II infects only Nambu, and pathotype III infects all three cultivars (Ohki et al. 2014; Ohto et al. 2006). In a comparison of predicted amino acid sequences among 14 isolates from various places in Japan, WYMV comprised three genotypes for viral RNA1 and two for viral RNA2 (Ohki et al. 2014). Because the viral RNA1 genotypes were correlated with the pathotypes, a viral factor or factors associated

†Corresponding author: T. Ohki; take2001@affrc.go.jp

*The $\boldsymbol{e}$-Xtra logo stands for "electronic extra" and indicates that one supplementary table is published online.

The author(s) declare no conflict of interest.

(c) 2019 The American Phytopathological Society with pathogenicity is suggested to be present in viral RNA1 (Ohki et al. 2014). On the other hand, the genetic background of wheat cultivars that are resistant to WYMV is currently unknown, although some genes conferring resistance to WYMV have been reported (Liu et al. 2005; Nishio et al. 2010; Suzuki et al. 2015; Zhu et al. 2012). Therefore, the relationship between pathotypes and resistance genes remains unclear for WYMV.

To understand the relationship between WYMV pathotypes and wheat resistance genes, it is important to elucidate a viral factor that is associated with the pathogenicity. Thus, in this study, we examined genomic regions involved in the pathogenicity of WYMV using chimeric and site-directed mutants. Our results revealed that the differential pathogenicity between pathotypes I and II of WYMV is determined by the $\mathrm{N}$-terminal region of $\mathrm{CI}$ protein and is likely dependent on the ability of cell-to-cell or long-distance viral movement, in which the CI protein is involved.

\section{MATERIALS AND METHODS}

Plants. Wheat (T. aestivum L.) cultivars Fukuho, Shirane, Nambu, and Hokkai 240 were used in this study. Wheat plants were cultivated in the commercial soil Engai-baido (Hokuren, Hokkaido, Japan) with the fertilizer Aid-ball Ca (Sumitomo Chemical Garden Products, Tokyo, Japan) in 9-cm plastic pots.

Construction of infectious cDNA clones of WYMV RNA1 and RNA2. Viral full-length cDNA clones were constructed from total RNA isolated from wheat leaves infected with WYMV-Su (pathotype I) and WYMV-Ch (pathotype II) obtained from Suzuka, Mie, and Chitose, Hokkaido, respectively (Ohki et al. 2014). Total RNA was extracted using an RNeasy Plant Mini Kit (Qiagen, Valencia, CA, U.S.A.). Primers used for constructing full-length cDNA clones and chimeric and site-directed mutants are summarized in Table 1 and Supplementary Table S1. First-strand cDNA was synthesized from $0.5 \mu \mathrm{g}$ of RNA using PrimeScript Reverse transcription (Takara Bio, Shiga, Japan) and specific primers, in 
accordance with the manufacturer's protocols. The cDNA reactions were treated with $40 \mathrm{U}$ of RNase $\mathrm{H}$ (Takara Bio) at $37^{\circ} \mathrm{C}$ for $20 \mathrm{~min}$, followed by amplification of DNA fragments by KOD NEO Plus polymerase (Toyobo, Osaka, Japan). To construct the full-length cDNA clones for viral RNA1, two DNA fragments (RNA1-Fr1 and RNA1-Fr2) were amplified using oligonucleotides WR1-F1 and WR1-R1, and WR1-F2 and WR1-R2, respectively. Polymerase chain reaction (PCR) conditions consisted of initial denaturation at $94^{\circ} \mathrm{C}$ for $2 \mathrm{~min}$, followed by 35 cycles of denaturation at $98^{\circ} \mathrm{C}$ for $10 \mathrm{~s}$, annealing at $55^{\circ} \mathrm{C}$ for $30 \mathrm{~s}$, and extension at $68^{\circ} \mathrm{C}$ for $2 \mathrm{~min}$. After digestion with Pst + NarI for RNA1-Fr1 and NarI + XmaI for RNA1-Fr2, the two fragments were ligated into pUC19 digested with Pst $\mathrm{I}+X m a \mathrm{I}$ using DNA Ligation Kit Mighty Mix (Takara Bio), then used to transform Escherichia coli strain JM109. To construct the full-length cDNA clones for viral RNA2, a DNA fragment was amplified using oligonucleotides WR2-F1 and WR2-R1. After digestion with $B a m \mathrm{HI}+X m a \mathrm{I}$, the PCR-amplified fragment was similarly ligated into pUC19. The T7 RNA polymerase sequence and a unique $X m a I$ restriction site were engineered at the $5^{\prime}$ and $3^{\prime}$ ends of viral genomic sequences of RNA1 and RNA2, respectively, to synthesize in vitro RNA transcripts.

Construction of chimeric and site-directed mutants. Chimeric mutants of WR1-Su/Ch, WR1-Ch/Su, WR1-Ch-Su/ ChCI, and WR1-Su-Ch/SuCI were constructed using NarI (nucleotide 4,393) and SnaBI (nucleotide 1,955) restriction enzyme sites (Fig. 1). To construct the chimeric mutants WR1-Ch-SuCI and WR1-Su-ChCI (Fig. 1), the fragment of CI cistron was amplified by PCR using oligonucleotides FuCI-Ins-F ( $\mathrm{Su}$ ) and FuCI-Ins-R from WR1-Su, and oligonucleotides FuCI-Ins-F (Ch) and FuCI-Ins-R from WR1-Ch. The approximately $10-\mathrm{kbp}$ vector fragment including pUC19 was also amplified by inverse PCR using the oligonucleotides FuCI-Vec-F and FuCI-Vec-R from WR1-Su or WR1-Ch. After purification by gel extraction, the DNA fragments were ligated using an In-fusion HD cloning kit (Takara Bio) with 15-bp overlaps between the fragment of a CI cistron and a vector. Site-directed mutants were constructed using a KOD-PlusMutagenesis Kit (Toyobo) (Fig. 2). Linear DNA was amplified from clones by inverse PCR using primers containing the target base substitution at the $5^{\prime}$ end. After the PCR solution was treated with DpnI to remove the template plasmid, $2 \mu$ l of PCR solution, $5 \mu \mathrm{l}$ of Ligation High (Toyobo), $1 \mu \mathrm{l}$ of T4 Polynucleotide Kinase (Toyobo), and $7 \mu \mathrm{l}$ of distilled water were mixed, incubated at $16^{\circ} \mathrm{C}$ for more than $1 \mathrm{~h}$, and used to transform E. coli strain JM109. The sequences of all clones were confirmed using a 3130 Genetic Analyzer (Thermo Fisher Scientific, Waltham, MA, U.S.A.) and BigDye terminator v3.1 (Thermo Fisher Scientific).

Inoculation of wheat seedlings with in vitro transcripts. Plasmids were linearized with $\mathrm{XmaI}$, a unique restriction enzyme site engineered at the $3^{\prime}$ end of the viral genome sequence, and were purified using phenol-chloroform and a FastGene Gel/PCR Extraction Kit (Nippon Genetics, Tokyo, Japan). RNA transcripts with the cap analog were synthesized in vitro from $500 \mathrm{ng}$ of linear plasmid DNA using the T7 RiboMAX large-scale RNA production system (Promega Corp., Madison, WI, U.S.A.) and $\mathrm{m}^{7} \mathrm{G}\left(5^{\prime}\right) \mathrm{ppp}\left(5^{\prime}\right)$ G RNA Cap Structure Analog (New England Biolabs, Ipswich, MA,
U.S.A.), in accordance with the manufacturers' instructions. In vitro transcription mixtures of RNA1 and RNA2 were diluted 1:5 with distilled water, then rubbed onto carborundum-dusted wheat

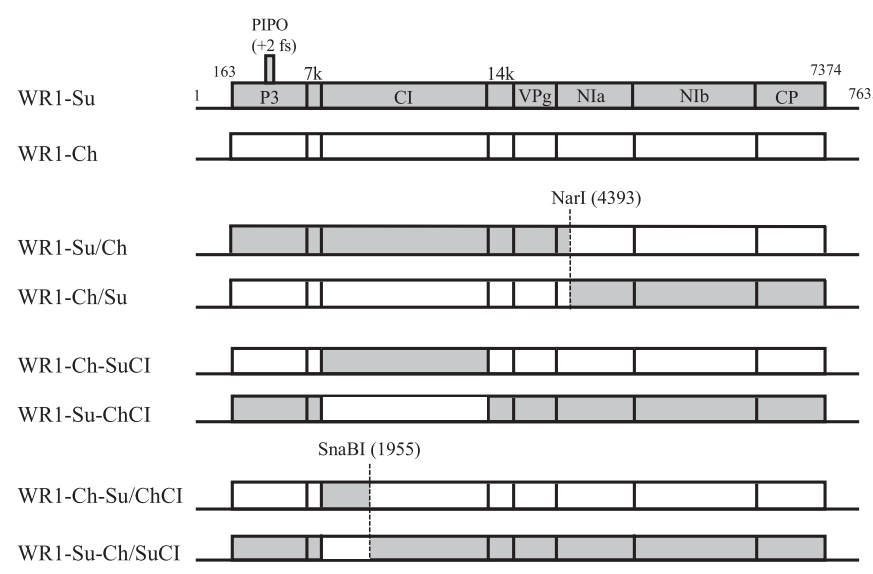

Fig. 1. Schematic representation of chimeric constructs between full-length cDNA clones WR1-Su and WR1-Ch. Gray box represents the sequence of WR1-Su; open box represents the sequence of WR1-Ch. The dotted line indicates the position of the restriction enzyme site (nucleotides) in the wheat yellow mosaic virus RNA1 genomic sequence. The short open reading frame, termed PIPO, is shown only for WR1-Su.

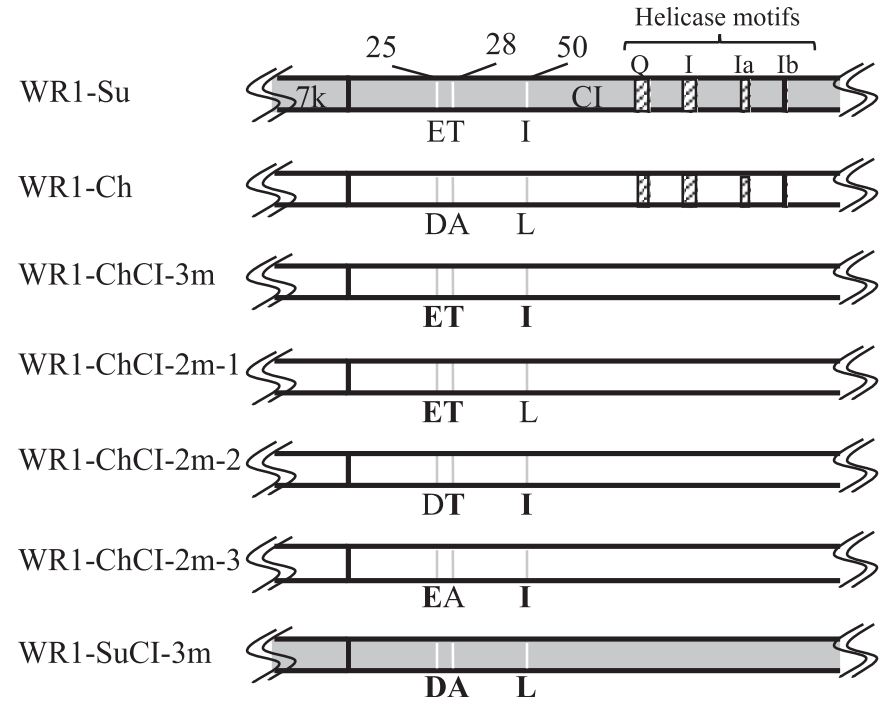

Fig. 2. Schematic representation of point-mutated constructs from full-length cDNA clones of WR1-Su and WR1-Ch. Gray box represents the sequence of WR1-Su; open box represents the sequence of WR1-Ch. Gray and white lines indicate the position of an amino-acid substitution on the N-terminal region of cylindrical inclusion protein. Bold letters indicate substituted amino acids. Putative motifs conserved among superfamily 2 RNA helicases (Kadaré and Haenni 1997) are shown as shaded boxes and only for WR1-Su and WR1-Ch.

TABLE 1. Primers for construction of full-length cDNA clones of wheat yellow mosaic virus

\begin{tabular}{|c|c|c|c|c|}
\hline Primer & Sequence & Direction & Position & Notes \\
\hline WR1-F1 & $\begin{array}{l}\text { atat } C T G C A G \\
\text { TAATACGACTCACTATAGAAAATAAAACCACCACAGACC }\end{array}$ & Forward & $1-21$ & $\begin{array}{l}\text { Italics: PstI recognition sequence; } \\
\text { underline: T7 promoter }\end{array}$ \\
\hline WR1-R1 & ATTGCCTCGAGAATGTTGCT & Reverse & $3,883-3,902$ & \\
\hline WR1-F2 & GGGAGGTGACCAAGGATATG & Forward & $3,602-3,621$ & \\
\hline WR1-R2 & atat $G G G C C C$ T(42)GTTACCTTCTGGTACTCGTA & Reverse & $7,616-7,635$ & Italics: $X m a I$ recognition sequence \\
\hline WR2-F1 & $\begin{array}{l}\text { atat } G G A T C C \\
\text { TAATACGACTCACTATAGAAAAATAAAACCACCACAAAC }\end{array}$ & Forward & $1-21$ & $\begin{array}{l}\text { Italics: Bam HI recognition sequence; } \\
\text { underline: T7 promoter }\end{array}$ \\
\hline WR2-R1 & atat $C C C G G G$ T(42)GTCACATTTCCTGTGTACAA & Reverse & $3,632-3,651$ & Italics: $X m a$ I recognition sequence \\
\hline
\end{tabular}


seedlings Shirane at the 2.5- to 3-leaf stage. Infectivity of wheat seedlings by recombinant viruses was examined by enzyme-linked immunosorbent assay (ELISA), infected leaves of Shirane were ground in approximately 10 volumes (vol/wt) of $50 \mathrm{mM}$ phosphate buffer ( $\mathrm{pH}$ 8.0) and the sap was used as inoculum in the subsequent experiments. Inoculated wheat seedlings were cured at $8^{\circ} \mathrm{C}$ for 5 days in the dark, followed by at $10^{\circ} \mathrm{C}$ in a growth chamber with a 14-h photoperiod (approximately 10,000 lux). At 6 weeks after inoculation, WYMV was detected from the second unfolded leaf (a noninoculated upper leaf) by double-antibody sandwich ELISA (Netsu et al. 2011). Samples were considered positive for virus infection if absorbance at $405 \mathrm{~nm}$ was two times greater than that of mock-inoculated leaves.

Inoculation of wheat mesophyll protoplasts with in vitro transcripts. Wheat mesophyll protoplasts were prepared and inoculated with in vitro transcripts in accordance with the procedure of Ohsato et al. (2003), with minor modifications. Seedlings of Fukuho and Shirane wheat were grown in commercial soil (Engeibaido) at $22^{\circ} \mathrm{C}$ with a $14-\mathrm{h}$ photoperiod in a growth cabinet for 7 days. After an approximately 3 -cm leaf section was cut from the middle of the leaf, the epidermis was carefully peeled off, followed by immediate immersion in an enzyme solution consisting of 0.65 M mannitol, 0.2\% Cellulase Onozuka RS (Yakult, Tokyo, Japan), 0.07\% Macerozyme R-10 (Yakult), and $0.1 \%$ bovine serum albumin at $\mathrm{pH}$ 5.7. Incubation was then performed without stirring at $30^{\circ} \mathrm{C}$ for $3 \mathrm{~h}$ in the dark. The protoplasts were collected by centrifugation in a swing rotor at $70 \times g$ for $5 \mathrm{~min}$, and washed with $0.65 \mathrm{M}$ mannitol containing $10 \mathrm{mM} \mathrm{CaCl}_{2}$. Protoplasts $\left(1.5 \times 10^{5}\right)$ were transfected with a mixture of RNA1 and RNA2 transcripts from $40 \mathrm{ng}$ of each linear DNA using polyethylene glycol solution (Ohsato et al. 2003). Protoplasts were washed twice with $0.65 \mathrm{M}$ mannitol containing $10 \mathrm{mM} \mathrm{CaCl}_{2}$ and cultured in $1 \mathrm{ml}$ of medium $\left(0.2 \mathrm{mM} \mathrm{KH}_{2} \mathrm{PO}_{4}, 1 \mathrm{mM} \mathrm{KNO}_{3}, 1 \mathrm{mM} \mathrm{MgSO}_{4}, 1 \mathrm{mM} \mathrm{KI}, 0.1 \mathrm{mM}\right.$

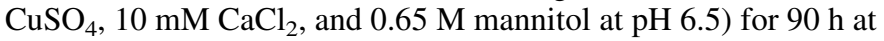
$10^{\circ} \mathrm{C}$ in the dark.
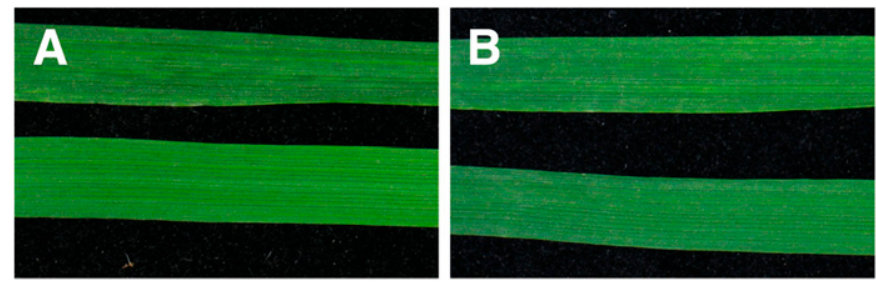

Fig. 3. Symptoms on upper uninoculated leaves of Shirane wheat at 4 weeks after inoculation. A, Mild mosaic symptoms on leaves inoculated with in vitro transcripts from full-length cDNA clones WR1-Su and WR2-Su. B, Mild mosaic symptoms after inoculation with in vitro transcripts from full-length cDNA clones WR1-Ch and WR2-Ch. Top, infected leaf; bottom, mock-inoculated leaf.
Western blot analysis. Collected protoplasts were suspended in $50 \mu$ l of Laemmli sample buffer (Bio-Rad, Hercules, CA, U.S.A.) containing $0.1 \mathrm{M}$ dithiothreitol and $1 \times$ Complete Protease Inhibitor Cocktail (Merck, Darmstadt, Germany) and heated at $95^{\circ} \mathrm{C}$ for $5 \mathrm{~min}$; then, $5 \mu \mathrm{l}$ of the sample was loaded per lane in a $15 \%$ sodium dodecyl sulfate polyacrylamide gel. After electrophoresis, proteins were transferred to Hybond-P membrane (GE Healthcare, Chicago, IL, U.S.A.) by semidry blotting and the membrane was blocked with Tris buffered saline with Tween 20 containing 5\% skim milk. The membrane was subjected to anti-WYMV CP immunoglobulin G (IgG) for $1 \mathrm{~h}$, followed by goat antirabbit IgG conjugated to alkaline phosphatase (Merck) for $1 \mathrm{~h}$, and developed with the BCIP/NBT liquid substrate system (Merck). Images were scanned from membranes at 600 dpi using a flatbed scanner, and the density of bands was quantified using ImageJ (National Institutes of Health, Bethesda, MD, U.S.A.; https://imagej.nih.gov/ij/index.html). The intensity of the $\mathrm{CP}$ band of each sample was normalized with the intensity of the rubisco large subunit band of the corresponding sample stained with QuickBlue Staining Solution (BioDynamics Laboratory, Tokyo, Japan). Statistical analyses ( $t$ test) were performed with EZR, a graphical user interface for R (Kanda 2013).

\section{RESULTS}

Systemic infection of wheat by in vitro transcripts from full-length cDNA clones. Two full-length cDNA clones, pWR1$\mathrm{Su}$ (DDBJ accession LC404158) for viral RNA1 and pWR2-Su (LC404160) for viral RNA2, were constructed from total RNA isolated from wheat leaves infected with WYMV-Su. Similarly, two full-length cDNA clones from WYMV-Ch-infected leaves were constructed and named pWR1-Ch (LC404159) and pWR2-Ch (LC404161) for RNA1 and RNA2, respectively. The 11 amino acid changes in RNA1 and 12 amino acid changes in RNA2 that were reported previously between WYMV-Su and WYMV-Ch (Ohki et al. 2014) were all conserved in cDNA clones. In vitro transcripts of WR1-Su + WR2-Su or WR1-Ch + WR2-Ch elicited mild mosaic symptoms on upper uninoculated leaves of Shirane wheat at 4 weeks postinoculation, similar to those elicited by the wild-type viruses (Fig. 3). In Western blot analysis, a 32-kDa CP was detected from WYMV-Su- or WYMV-Ch-infected leaves (data not shown).

Crude sap from leaves infected with the wild-type and reassortant viruses was used to inoculate Fukuho, Nambu, Shirane, and Hokkai240 (Table 2). The recombinant WYMV-Su from pWR1$\mathrm{Su}+\mathrm{pWR} 2-\mathrm{Su}$ cDNA clones was detected in upper uninoculated leaves of all cultivars except Hokkai 240, thus indicating that WYMV-Su's pathogenicity can be categorized as pathotype I. In contrast, the recombinant virus from cDNA clones, pWR1-Ch + pWR2-Ch, infected Nambu and Shirane but not Fukuho and Hokkai240, thus indicating that WYMV-Ch's pathogenicity can be categorized as pathotype II. These results were similar to those reported for the wild-type viruses (Ohki et al. 2014; Ohto et al.

TABLE 2. Systemic infection of four wheat cultivars by wild-type and reassortant viruses

\begin{tabular}{|c|c|c|c|c|c|c|}
\hline \multirow[b]{2}{*}{ Virus (RNA1, RNA2) } & \multirow[b]{2}{*}{$\operatorname{Exp}^{b}$} & \multicolumn{4}{|c|}{ Number of infected plants ${ }^{a}$} & \multirow[b]{2}{*}{ Pathotype } \\
\hline & & Fukuho & Nambu & Shirane & Hokkai 240 & \\
\hline \multirow[t]{3}{*}{ WR1-Su, WR2-Su } & 1 & $7 / 13$ & - & $6 / 10$ & $0 / 12$ & $\mathrm{I}$ \\
\hline & 2 & $18 / 25$ & - & $17 / 23$ & - & - \\
\hline & 3 & - & $7 / 23$ & $14 / 19$ & - & - \\
\hline \multirow[t]{3}{*}{ WR1-Ch, WR2-Ch } & 1 & $0 / 14$ & - & $10 / 12$ & $0 / 16$ & II \\
\hline & 2 & $0 / 25$ & - & $24 / 25$ & - & - \\
\hline & 3 & - & $12 / 19$ & $21 / 21$ & - & - \\
\hline \multirow[t]{2}{*}{ WR1-Su, WR2-Ch } & 1 & $16 / 21$ & - & $25 / 26$ & - & $\mathrm{I}$ \\
\hline & 2 & - & $17 / 20$ & $21 / 21$ & - & - \\
\hline \multirow[t]{2}{*}{ WR1-Ch, WR2-Su } & 1 & $0 / 27$ & - & $24 / 27$ & - & II \\
\hline & 2 & - & $10 / 19$ & $12 / 22$ & - & - \\
\hline
\end{tabular}

a Infected plants/tested plants; - = not tested.

b Experiment. 
2006). When wheat cultivars were inoculated with the reassortant viruses between RNA1 and RNA2, WR1-Su + WR2-Ch infected Fukuho, Nambu, and Shirane but WR1-Ch + WR2-Su infected Shirane and Nambu but not Fukuho. These results revealed that WYMV RNA1 possesses viral determinants responsible for systemically infecting Fukuho wheat.

The CI protein harbors determinants responsible for differential infection of Fukuho by WYMV pathotypes. Chimeric mutants were used to map viral determinants responsible for differential pathogenicity of WYMV pathotypes on Fukuho. In vitro transcripts of chimeric viruses between RNA1 genomes of WR1-Su and WR1-Ch (Fig. 1) were inoculated onto Fukuho (resistant to pathotype II) and Shirane (susceptible). WR1-Su/Ch, possessing nucleotides 1 to 4,393 from WR1-Su and nucleotides 4,394 to 7,635 from WR1-Ch, systemically infected both wheat cultivars. However, a hybrid virus, WR1-Ch/Su, possessing nucleotides 1 to 4,395 from WR1-Ch and the remaining sequence from WR1-Su infected only the susceptible wheat Shirane (Table 3). In a previous analysis, many of the amino-acid substitutions between pathotypes I and II were found in the CI protein at the $5^{\prime}$-terminal region of viral RNA1 (Ohki et al. 2014). Therefore, WR1-Ch-SuCI and WR1-Su-ChCI were created by precisely replacing the CI cistron with that of WR1-Su and WR1$\mathrm{Ch}$, respectively. WR1-Ch-SuCI having the CI cistron from WR1Su systemically infected Fukuho but WR1-Su-ChCI with the CI of WR1-Ch failed to infect Fukuho at detectable levels (Table 3). Furthermore, WR1-Ch-Su/ChCI, having the N-terminal region of CI cistron from WR1-Su, systemically infected Fukuho (Table 3). These results indicated that the $\mathrm{N}$-terminal three differential amino

TABLE 3. Systemic infection in Fukuho and Shirane by chimeric viruses

\begin{tabular}{|c|c|c|c|c|c|}
\hline \multicolumn{2}{|l|}{ Virus } & \multirow[b]{2}{*}{$\operatorname{Exp}^{b}$} & \multicolumn{2}{|c|}{$\begin{array}{l}\text { Number of } \\
\text { infected plants }\end{array}$} & \multirow[b]{2}{*}{ Pathotype } \\
\hline RNA1 & RNA2 & & Fukuho & Shirane & \\
\hline WRl-Su/Ch & WR2-Su & 1 & $25 / 25$ & $26 / 26$ & I \\
\hline WRl-Ch/Su & WR2-Su & 1 & $0 / 27$ & $2 / 27$ & II \\
\hline \multirow[t]{2}{*}{ WR1-Ch-SuCI } & WR2-Su & 1 & $6 / 29$ & $14 / 30$ & I \\
\hline & - & 2 & $22 / 32$ & $6 / 13$ & - \\
\hline \multirow[t]{2}{*}{ WR1-Su-ChCI } & WR2-Su & 1 & $0 / 25$ & $23 / 28$ & II \\
\hline & - & 2 & $0 / 25$ & $7 / 15$ & - \\
\hline WR1-Ch-Su/ChCI & WR2-Su & 1 & $10 / 23$ & $9 / 24$ & I \\
\hline \multirow[t]{2}{*}{ WR1-Su-Ch/SuCI } & WR2-Su & 1 & $0 / 27$ & $24 / 27$ & II \\
\hline & - & 2 & $0 / 35$ & $5 / 25$ & - \\
\hline
\end{tabular}

a Infected plants/tested plants.

b Experiment.

TABLE 4. Systemic infection in Fukuho and Shirane by site-directed mutants

\begin{tabular}{|c|c|c|c|c|c|}
\hline \multicolumn{2}{|l|}{ Virus } & \multirow[b]{2}{*}{$\operatorname{Exp}^{\mathrm{b}}$} & \multicolumn{2}{|c|}{$\begin{array}{l}\text { Number of } \\
\text { infected plants }\end{array}$} & \multirow[b]{2}{*}{ Pathotype } \\
\hline RNA1 & RNA2 & & Fukuho & Shirane & \\
\hline \multirow[t]{2}{*}{ WR1-ChCI-3m } & WR2-Su & 1 & $1 / 12$ & $1 / 11$ & $\mathrm{I}$ \\
\hline & - & 2 & $5 / 26$ & $4 / 27$ & - \\
\hline \multirow[t]{2}{*}{ WR1-ChCI-3m } & WR2-Ch & 1 & $3 / 12$ & $10 / 13$ & I \\
\hline & - & 2 & $11 / 28$ & $12 / 27$ & - \\
\hline \multirow[t]{2}{*}{ WR1-ChCI-2m-1 } & WR2-Ch & 1 & $0 / 23$ & $11 / 17$ & II \\
\hline & - & 2 & $0 / 24$ & $6 / 23$ & - \\
\hline \multirow[t]{2}{*}{ WR1-ChCI-2m-2 } & WR2-Ch & 1 & $0 / 23$ & $17 / 20$ & II \\
\hline & - & 2 & $0 / 20$ & $14 / 16$ & - \\
\hline \multirow[t]{2}{*}{ WR1-ChCI-2m-3 } & WR2-Ch & 1 & $0 / 24$ & $13 / 24$ & II \\
\hline & - & 2 & $0 / 19$ & $5 / 19$ & - \\
\hline \multirow[t]{2}{*}{ WR1-SuCI-3m } & WR2-Su & 1 & $0 / 24$ & $19 / 23$ & II \\
\hline & - & 2 & $0 / 24$ & $21 / 24$ & - \\
\hline \multirow[t]{2}{*}{ WR1-SuCI-3m } & WR2-Ch & 1 & $1 / 24$ & $23 / 24$ & Intermediate type ${ }^{c}$ \\
\hline & - & 2 & $0 / 24$ & $4 / 24$ & - \\
\hline
\end{tabular}

a Infected plants/tested plants.

b Experiment.

c Systemic infection in Fukuho was rare. acids in the CI cistron of WR1-Su from those of WR1-Ch are most likely responsible for systemic infection of Fukuho.

Site-directed mutagenesis was employed to mutate two of three or all three differential amino acids in WR1-Ch similarly to those in WR1-Su and tested their infectivity on Fukuho and Shirane (Fig. 2; Table 4). The results showed that WR1-ChCI-3m, created by the introduction of all three amino acids in WR1-Ch as in WR1-Su, systemically infected Fukuho (Table 4). The authenticity of the virus sequence in upper uninoculated leaves was confirmed by direct sequencing and found that it was identical to the sequence of WR1-ChCI-3m. No systemic infection was observed in Fukuho plants inoculated with WR1-Ch mutants harboring two of three differed amino acids, as in WR1-Su. Conversely, WR1-SuCI-3m, created by substitution of three differed amino acids in WR1-Su as in WR1-Ch, systemically infected Fukuho at drastically reduced rates (Table 4). These results indicated that the three different amino acids at the N-terminal region of CI protein between WYMVSu and -Ch pathotypes (Fig. 2) are involved in differential pathogenicity to Fukuho by WYMV pathotypes.

WYMV-Ch replicates in Fukuho mesophyll protoplasts. We next examined whether failure to infect Fukuho by WYMV-Ch is due to its incompatibility at the viral replication or movement level. Replication of WYMV-Su and -Ch were examined in mesophyll protoplasts isolated from Fukuho and Shirane leaves. Mesophyll protoplasts were inoculated with a mixture of in vitro transcripts of RNA1 and RNA2 from WR1-Su, WR2-Su, WR1-Ch, or WR2-Ch in different combinations. The levels of CP accumulation in mesophyll protoplasts of Fukuho and Shirane was examined at $90 \mathrm{~h}$ postinoculation by Western blot analysis (Fig. 4A). Our data revealed that viral CP accumulated in Fukuho and Shirane protoplasts inoculated with all combinations of RNA1 and 2 (Fig. 4A), although WR1-Ch + WR2-Ch and WR1-Ch + WR2-Su did not infect Fukuho (Table 2). The level of CP accumulation in WR1-Su + WR2-Su- or WR1-Ch + WR2-Ch-inoculated Fukuho protoplasts was compared and indicated that $\mathrm{CP}$ accumulation levels did not differ significantly $(t$-test; $P<0.05)$ (Fig. 4B). These

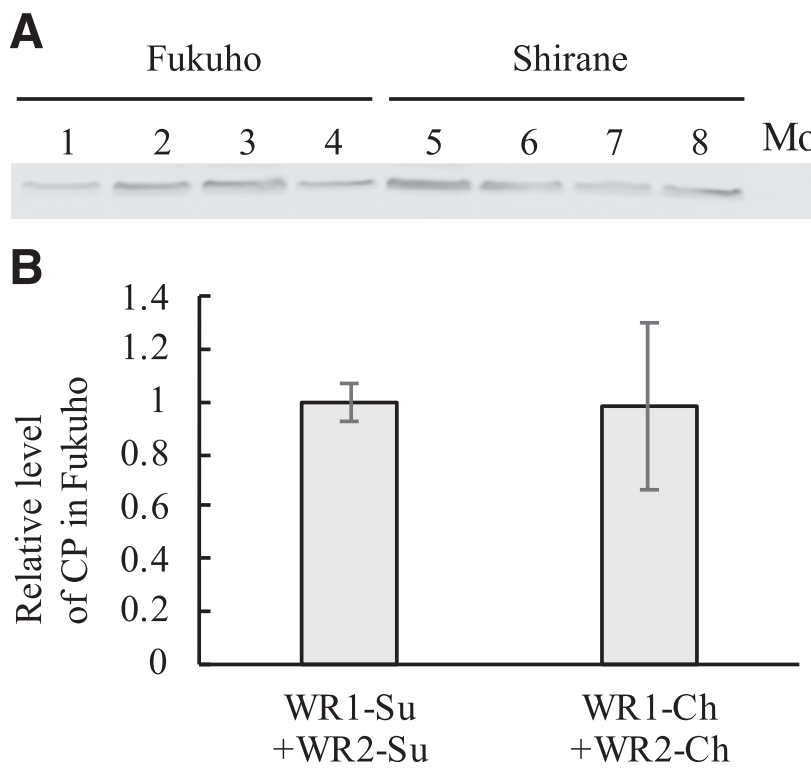

Fig. 4. Western blot analysis of the coat protein (CP) of wheat yellow mosaic virus from mesophyll protoplasts of Fukuho and Shirane. A, Detection of CP in protoplasts inoculated with a mixture of in vitro transcripts from full-length cDNA clones. Lanes 1 and 5: WR1-Su + WR2-Su; lanes 2 and 6: WR1-Ch + WR2-Ch; lanes 3 and 7: WR1-Su + WR2-Ch; and lanes 4 and 8: WR1-Ch + WR2-Su. B, Relative accumulation of CP of WR1-Su + WR2-Su and WR1-Ch + WR2-Ch in protoplasts of Fukuho. The relative scores were calculated against the average value of WR1-Su + WR2-Su. The experiment was replicated four times. 
data revealed that a difference in pathogenicity in Fukuho between WYMV pathotypes I and II is not associated with viral replication at the cellular level.

\section{DISCUSSION}

The three pathotypes of WYMV present a clear regional distribution in Japan: pathotype II is found in northern areas, pathotype I in central areas, and pathotype III in southern areas of the country (Ohki et al. 2014; Ohto et al. 2006). Of these three pathotypes of WYMV, pathotypes I and II are the primary ones in Japan. The pathotype I of WYMV infects Fukuho systemically but pathotype II failed to infect this wheat cultivar (Ohki et al. 2014; Ohto et al. 2006). However, the mechanism underlying the differential pathogenicity between these two pathotypes is not known. In this study, we demonstrated that three amino-acid substitutions at the $\mathrm{N}$-terminal region of the $\mathrm{CI}$ protein are involved in infectivity to Fukuho. Because there was no difference in viral replication at the single-cell level, the difference in pathogenicity between pathotypes I and II should depend on the ability of the virus to move cell to cell or long distance in Fukuho. In terms of studies on bymoviruses, including WYMV, this is the first report to reveal the involvement of a bymoviral CI protein in differential pathogenicity.

Potyviral CI protein is known to have multiple functions (Sorel et al. 2014). Of these, NTPase and RNA helicase activities of CI protein are indispensable for viral replication (Fernández et al. 1997). However, the three amino-acid substitutions in the CI protein of WYMV were not located in the conserved motifs of superfamily 2 RNA helicases, to which bymoviral CI proteins belong (Kadaré and Haenni 1997) (Fig. 2). Furthermore, the amount of CP accumulated did not differ between WYMV-Su (pathotype I) and WYMV-Ch (pathotype II) in Fukuho (Fig. 4), suggesting that these substitutions are not associated with NTPase and RNA helicase activity and do not affect viral replication.

The CI protein is also required for virus movement within the plant (Sorel et al. 2014). The ultrastructural analyses showed that the CI protein forms cone-shaped structures close to the plasmodesmata through which viruses move between plant cells, and potyviral CPs are associated with these cone-shaped structures (Calder and Ingerfeld 1990; Lawson and Hearon 1971; Roberts et al. 1998; Rodríguez-Cerezo et al. 1997). The CI protein has also been detected in the phloem and companion cells (Lawson and Hearon 1971; Otulak and Garbaczewska 2012). In Wheat spindle streak mosaic virus, which is closely related to WYMV and which causes similar yellow mosaic disease of wheat in Europe and Canada (Namba et al. 1998; Xiaoyun et al. 1998), CI protein is also localized near the plasmodesmata (Langenberg 1993), and CP binds the CI protein (Langenberg 1986). Therefore, CI protein of WYMV is also considered to be involved in viral movement. Reversegenetics analysis showed that some mutations in the $\mathrm{N}$-terminal region of CI protein can result in a delay or block of viral movement in potyviruses without affecting RNA helicase activity or virus replication (Carrington et al. 1998; Gómez de Cedrón et al. 2006). The mutations in this region reduce the binding strength of CI protein self-interaction in the yeast two-hybrid system, suggesting that CI-CI interactions are required for viral movement (Gómez de Cedrón et al. 2006). Therefore, it is possible that the three aminoacid substitutions in the N-terminal region of WYMV CI protein may also affect the self-interaction.

Among genes conferring resistance to WYMV located on the 2DL, 5AL, and 3BS chromosomes of wheat (Liu et al. 2005; Nishio et al. 2010; Suzuki et al. 2015; Zhu et al. 2012), the resistance gene on chromosome 3BS was detected in Fukuho but not in Shirane from an analysis of DNA markers (Kojima et al. 2019). Interestingly, wheat cultivars having the resistance gene on chromosome 3BS was partially effective against pathotype II in the field trial (Kojima et al. 2019). Therefore, the 3BS gene is likely to be involved in blocking the viral movement of pathotype II. For further analysis, identification of the 3BS gene is necessary.

Some genes of host plants conferring resistance against potyvirid species inhibit viral movement. The Wsml and Wsm2 genes in wheat block the long-distance viral movement of Wheat streak mosaic virus and Triticum mosaic virus (Tatineni et al. 2016). Additionally, the $b c-1$ and $b c-2$ genes in common bean (Phaseolus vulgaris) affect the systemic spread of Bean common mosaic necrosis virus (Feng et al. 2017) and Bean common mosaic virus (Feng et al. 2018) without affecting replication and cell-to-cell movement. Moreover, barley cultivars possessing rym 2 or rym 3 are resistant to Barley yellow mosaic virus isolate JK05; however, they permit viral replication at the single-cell level (You and Shirako 2013). The viral proteins involved in these resistance have yet to be identified. On the other hand, Soybean mosaic virus strain G7 (SMV-G7) was shown to be restricted to inoculated leaves of Rsv3genotype soybean, suggesting that the $R s v 3$ resistance gene restricts viral movement (Zhang et al. 2009). Interestingly, the CI protein of SMV determines the virulence to Rsv3-genotype soybean; a single amino-acid substitution in the C-terminal region for SMV-G7H (Seo et al. 2009) and both N- and C-terminal regions for SMV-G7 (Zhang et al. 2009) are involved in the gain or loss of virulence. Although there are a few reports describing the CI protein as involved in resistance to viral movement, this protein should receive more attention as a viral factor involved in resistance.

As mentioned earlier, of the three WYMV pathotypes in Japan, pathotype III is found in southern Japan (Ohki et al. 2014; Ohto et al. 2006). The genomic sequences of pathotypes I and III are similar (Ohki et al. 2014). Pathotype III may be a strain that was derived from a change in pathotype I because wheat cultivars tolerant to pathotype I have been cultivated in southern Japan. As with pathotypes I and II, several amino-acid changes have been found in RNA1 between pathotype III and the other pathotypes (Ohki et al. 2014). To breed effective resistant cultivars, the pathogenicity of WYMV isolates and its relationship to host resistance genes in Japan need to be clarified.

\section{ACKNOWLEDGMENTS}

We thank Y. Shirako and H. Li of The University of Tokyo for technical support in preparing the wheat protoplasts and inoculation with in vitro transcripts.

\section{LITERATURE CITED}

Adams, M. J., Zerbini, F. M., French, R., Rabenstein, F., Stenger, D. C., and Valkonen, J. P. T. 2012. Bymovirus. Pages 1084-1086 in: Virus Taxonomy. Ninth Report of the International Committee on Taxonomy of Viruses. A. M. Q. King, M. J. Adams, E. B. Carstens, and E. J. Lefkowitz, eds. Elsevier/Academic Press, San Diego, CA, U.S.A.

Calder, V. L., and Ingerfeld, M. 1990. The roles of the cylindrical inclusion protein of a potyvirus in the induction of vesicles and in cell-to-cell spread. J. Struct. Biol. 105:62-66.

Carrington, J. C., Jensen, P. E., and Schaad, M. C. 1998. Genetic evidence for an essential role for potyviral CI protein in cell-to-cell movement. Plant J. 14:393-400.

Chung, B. Y.-W., Miller, W. A., Atkins, J. F., and Firth, A. E. 2008. An overlapping essential gene in the Potyviridae. Proc. Natl. Acad. Sci. U.S.A. 105:5897-5902.

Feng, X., Guzm, P., Myers, J. R., and Karasev, A. V. 2017. Resistance to Bean common mosaic necrosis virus conferred by the $b c-1$ gene affects systemic spread of the virus in common bean. Phytopathology 107:893-900.

Feng, X., Orellana, G. E., Myers, J. R., and Karasev, A. V. 2018. Recessive resistance to Bean common mosaic virus conferred by the bc-1 and bc-2 genes in common bean (Phaseolus vulgaris) affects long-distance movement of the virus. Phytopathology 108:1011-1018.

Fernández, A., Guo, H. S., Sáenz, P., Simón-Buela, L., Gómez de Cedrón, M., and García, J. A. 1997. The motif V of plum pox potyvirus CI RNA helicase is involved in NTP hydrolysis and is essential for virus RNA replication. Nucleic Acids Res. 25:4474-4480.

Gómez de Cedrón, M., Osaba, L., López, L., and García, J. A. 2006. Genetic analysis of the function of the plum pox virus CI RNA helicase in virus movement. Virus Res. 116:136-145. 
Han, C., Li, D., Xing, Y, Zhu, K., Tian, Z., Cai, Z., Yu, J., and Liu, Y. 2000. Wheat yellow mosaic virus widely occurring in wheat (Triticum aestivum) in China. Plant Dis. 84:627-630.

Inoue, T. 1969. Filamentous particles as the causal agent of yellow mosaic disease of wheat. Nougaku Kenkyu. 53:61-68.

Kadaré, G., and Haenni, A.-L. 1997. Virus-encoded RNA helicases. J. Virol. 71:2583-2590

Kanda, Y. 2013. Investigation of the freely available easy-to-use software 'EZR' for medical statistics. Bone Marrow Transplant. 48:452-458.

Kojima, H., Sasaya, T., Hatta, K., Seki, M., Oda, S., Kiribuchi-Otobe, C., Takayama, T., Fujita, Y., Chono, M., Matsunaka, H., and Nishio, Z. 2019. Response of Japanese wheat varieties to three pathotypes of wheat yellow mosaic virus. Euphytica 215:67. doi:10.1007/s10681-019-2387-4

Langenberg, W. G. 1986. Virus protein association with cylindrical inclusions of two viruses that infect wheat. J. Gen. Virol. 67:1161-1168.

Langenberg, W. G. 1993. The structural protein of three viruses in the potyviridae adhere only to their homologous cylindrical inclusions in mixed infections. J. Struct. Biol. 110:188-195.

Lawson, R. H., and Hearon, S. S. 1971. The association of pinwheel inclusions with plasmodesmata. Virology 44:454-456.

Liu, W., Nie, H., Wang, S., Li, X., He, Z., Han, C., Wang, J., Chen, X., Li, L., and Yu, J. 2005. Mapping a resistance gene in wheat cultivar Yangfu 9311 to yellow mosaic virus, using microsatellite markers. Theor. Appl. Genet. 111:651-657.

Namba, S., Kashiwazaki, S., Lu, X., Tamura, M., and Tsuchizaki, T. 1998. Complete nucleotide sequence of wheat yellow mosaic bymovirus genomic RNAs. Arch. Virol. 143:631-643.

Netsu, O., Ohki, T., Kojima, H., Oda, S., Aoki, E., Yoshioka, T., Yanagisawa, T., Ishikawa, K., and Sasaya, T. 2011. Development of serological diagnosis by ELISA for specific detection of four soil-borne viruses in wheat (Triticum aestivum) and barley (Hordeum vulgare). Annu. Rep. Kanto-Tosan Plant Prot. Soc. 58:13-17 (in Japanese).

Nishio, Z., Kojima, H., Hayata, A., Iriki, N., Tabiki, T., Ito, M., Yamauchi, H., and Murray, T. D. 2010. Mapping a gene conferring resistance to Wheat yellow mosaic virus in European winter wheat cultivar 'Ibis' (Triticum aestivum L.). Euphytica 176:223-229.

Ohki, T., Netsu, O., Kojima, H., Sakai, J., Onuki, M., Maoka, T., Shirako, Y., and Sasaya, T. 2014. Biological and genetic diversity of Wheat yellow mosaic virus (Genus Bymovirus). Phytopathology 104:313-319.

Ohsato, S., Miyanishi, M., and Shirako, Y. 2003. The optimal temperature for RNA replication in cells infected by Soil-borne wheat mosaic virus is $17^{\circ} \mathrm{C}$. J. Gen. Virol. 84:995-1000.

Ohto, Y., Hatta, K., and Ishiguro, K. 2006. Differential wheat cultivar to discriminate pathogenicity of Japanese Wheat yellow mosaic virus
(WYMV) isolates. Jpn. J. Phytopathol. 72:93-100 (in Japanese with English summary).

Otulak, K., and Garbaczewska, G. 2012. Cytopathological Potato virus Y structures during solanaceous plants infection. Micron 43:839-850.

Roberts, I. M., Wang, D., Findlay, K., and Maule, A. J. 1998. Ultrastructural and temporal observations of the potyvirus cylindrical inclusions (Cls) show that the $\mathrm{Cl}$ protein acts transiently in aiding virus movement. Virology 245: 173-181.

Rodríguez-Cerezo, E., Findlay, K., Shaw, J. G., Lomonossoff, G. P., Qiu, S. G., Linstead, P., Shanks, M., and Risco, C. 1997. The coat and cylindrical inclusion proteins of a potyvirus are associated with connections between plant cells. Virology 236:296-306.

Seo, J.-K., Lee, S.-H., and Kim, K.-H. 2009. Strain-specific cylindrical inclusion protein of Soybean mosaic virus elicits extreme resistance and a lethal systemic hypersensitive response in two resistant soybean cultivars. Mol. Plant-Microbe Interact. 22:1151-1159.

Sorel, M., Garcia, J. A., and German-Retana, S. 2014. The Potyviridae cylindrical inclusion helicase: A key multipartner and multifunctional protein. Mol. Plant-Microbe Interact. 27:215-226.

Suzuki, T., Murai, M. N., Hayashi, T., Nasuda, S., Yoshimura, Y., and Komatsuda, T. 2015. Resistance to Wheat yellow mosaic virus in Madsen wheat is controlled by two major complementary QTLs. Theor. Appl. Genet. 128:1569-1578.

Tatineni, S., Wosula, E. N., Bartels, M., Hein, G. L., and Graybosch, R. A. 2016. Temperature-dependent Wsm1 and Wsm2 gene-specific blockage of viral long-distance transport provides resistance to Wheat streak mosaic virus and Triticum mosaic virus in Wheat. Mol. Plant-Microbe Interact. 29: 724-738.

Xiaoyun, L., Kashiwazaki, S., Tamura, M., and Namba, S. 1998. The 3' terminal sequence of RNA1 of wheat spindle streak mosaic virus Canadian isolate (WSSMV-C). Eur. J. Plant Pathol. 104:765-768.

You, Y., and Shirako, Y. 2013. Evaluation of host resistance to Barley yellow mosaic virus infection at the cellular and whole-plant levels. Plant Pathol. 62:226-232.

Zhang, C., Hajimorad, M. R., Eggenberger, A. L., Tsang, S., Whitham, S. A., and Hill, J. H. 2009. Cytoplasmic inclusion cistron of Soybean mosaic virus serves as a virulence determinant on Rsv3-genotype soybean and a symptom determinant. Virology 391:240-248.

Zhu, X., Wang, H., Guo, J., Wu, Z., Cao, A., Bie, T., Nie, M., You, F. M., Cheng, Z., Xiao, J., Liu, Y., Cheng, S., Chen, P., and Wang, X. 2012. Mapping and validation of quantitative trait loci associated with wheat yellow mosaic bymovirus resistance in bread wheat. Theor. Appl. Genet. 124:177-188. 